\title{
Effects of alcohol intake in periodontitis progression in female rats: a histometric study
}

\author{
Daniela Martins de Souza ${ }^{1}$, Lucilene Hernandes Ricardo², Rosilene Fernandes da Rocha ${ }^{3}$ \\ ${ }^{1}$ Fundação Universitária Vida Cristã - Faculdade de Pindamonhangaba (FUNVIC/FAPI) - School of Dentistry, Department of Periodontology, \\ Pindamonhangaba, São Paulo, Brazil \\ 2Private Clinic, Pindamonhangaba, São Paulo, Brazil \\ ${ }^{3}$ Universidade Estadual Paulista - UNESP, São José dos Campos Dental School, São José dos Campos, São Paulo, Brazil
}

\begin{abstract}
Aim: To evaluate histometrically the effect of low and high caloric value of ethanol on ligatureinduced bone loss in female rats. Methods: Sixty female rats were divided into five groups of 12 animals each: Normal Control (water), Test A (low ethanol), Control A (low iso), Test B (high ethanol) and Control B (high iso). Control Groups A and B received diets with the same amount of calories consumed by Test Groups A and B, respectively, with ethanol replaced by isocaloric amounts of carbohydrate. Four weeks prior to the end of the experimental period, half of the rats in each group were randomly assigned to receive a ligature on mandibular molar, whereas the other half of group was left unligated. At 8 weeks, the animals were sacrificed, and the specimens were processed to obtain decalcified sections. The area of periodontal ligament and/or bone loss in the furcation region of the first molars was histometrically measured in five sections per specimen $\left(\mathrm{mm}^{2}\right)$. Results: Ethanol intake did not have effect on the alveolar bone loss in unligated teeth $(p>0.05)$. However, in ligature-induced periodontitis, high value of calories associated with ethanol feeding enhanced the area of bone loss $(p<0.05)$. Conclusions: The results of this study demonstrated that a low-ethanol diet did not affect the periodontium, while a high-ethanol diet may aggravate the progression of periodontitis, as demonstrated by the increased furcation region bone destruction in periodontal disease.
\end{abstract}

Keywords: ethanol; alveolar bone loss; periodontitis; rats.

\section{Introduction}

Periodontitis involves the destruction of supporting structures of the teeth, including the periodontal ligament, alveolar bone and gingival tissues ${ }^{1}$. However, some individuals are more susceptible than others to progressive periodontitis ${ }^{2}$. A large variety of factors exist that influence disease progression, including social and behavioral factors, systemic factors, genetic factors, tooth factors and microbial composition of the dental biofilm. Excessive alcohol consumption is considered

Received for publication: July 25, 2014 Accepted: September 16, 2014

Correspondence to: Daniela Martins de Souza Centro Clínico da Faculdade de Pindamonhangaba Rua Marechal Deodoro da Fonseca, 316, Centro CEP: 12 401-010, Pindamonhangaba/Brasil Phone: +55 12 3648-8323 E-mail: danimart.voy@terra.com.br; danimart.voy@gmail.com a behavioral factor that is associated with periodontal disease ${ }^{3}$.

Investigations have suggested that alcohol consumption is associated with increased severity of periodontitis and may be a risk indicator for periodontal disease ${ }^{4-5}$. However, more recent studies on humans have shown no relation between periodontal disease and alcohol consumption ${ }^{6-7}$.

The use of rat models has been applied in the evaluation of periodontal pathogenesis ${ }^{8}$ and regarding the influence of risk indicator/factors, such as estrogen deficiency ${ }^{9-10}$, alcohol consumption ${ }^{11-16}$, nicotine ${ }^{17}$ and simultaneous nicotine and alcohol use ${ }^{18}$ on disease progression. 
In the presence of experimental periodontitis, a high dose of ethanol (36\% of total calories in the diet) was associated with higher density of polymorphonuclear leukocytes, increasing the severity of periodontal inflammation in the ligature model of male rats ${ }^{15}$. Another study demonstrated that in male rats, ethanol increases ligature-induced bone loss when representing a low value $(22 \%)$ in total caloric value ${ }^{13}$.

Considering that more studies are necessary to test the hypothesis that alcohol consumption can aggravate destruction of the periodontium, the aim of this study was to evaluate histometrically the effect of low and high caloric values of ethanol consumption on alveolar bone loss associated with ligature-induced bone loss in female rats.

\section{Material and methods}

\section{Animals}

The Institutional Animal Research Committee at the University of São José dos Campos (São Paulo, Brazil) approved the protocol for all experimental (protocol $n^{\circ}$ 026/ 2003-PA/CEP).

Sixty adult female Wistar rats (4 months-old) weighing $270 \mathrm{~g}$ on average were randomized into five groups (12 rats/ group): Normal Control (water); Test A (low ethanol), composed of rats fed an ethanol-containing liquid diet with ethanol representing low caloric value; Control A (low iso), rats fed a pair-fed control diet (ethanol replaced by isocaloric amounts of carbohydrate); Test B (high ethanol), rats fed an ethanol-containing liquid diet with ethanol representing high caloric value; and Control B (high iso), rats fed a pair-fed control diet (ethanol replaced by isocaloric amounts of carbohydrate).

\section{Experimental procedures}

Prior to the experimental period, the rats comprising Test A and Test B groups were subjected to an adaptation period in which the ethanol concentration was increased until it reached experimental concentrations. A solution containing $5 \%$ ethanol was administered to ethanol Test A group for 7 days and ethanol Test B group received 5\% and 10\% ethanol for 7 days each. After this period, the rats received a diet containing the experimental concentrations for 8 weeks.

In Test A group, the rats received an ethanol solution (Ecibra, CETUS, Santo Amaro, SP, Brazil) at a concentration of $10 \%$ ethanol $\mathrm{v} / \mathrm{v}$ (by volume) and in Test B group, at a concentration of $20 \%$ ethanol $\mathrm{v} / \mathrm{v}$, in addition to standard rat chow (Guabi Nutrilador, Mogiana Alimentos, Campinas, SP, Brazil), both provided ad libitum.

The mean quantities of rat chow and ethanol solution consumed were calculated daily and one day after ethanol administration, the control $\mathrm{A}$ and $\mathrm{B}$ rats were fed an equal amount of rat chow as that consumed by the associated alcohol group and an equal volume of liquid diet, with the ethanol replaced by isocaloric amounts of carbohydrate.

Four weeks after beginning of experiment, general anesthesia was induced by intramuscular administration with solution of $13 \mathrm{mg} / \mathrm{kg}$ of $2 \%$ xylazine hydrochloride and $33 \mathrm{mg} /$ $\mathrm{kg}$ of ketamine hydrochloride. In order to induce periodontitis, cotton ligatures were placed around the cervix of the mandibular left first molar in six rats, leaving other six rats unligated to serve as a control.

The rats were sacrificed four weeks after ligature placement and their mandibles were removed and fixed in $10 \%$ neutral formalin for $48 \mathrm{~h}$. The left side of each mandible was submitted for histometric evaluation of area of periodontal ligament and/or bone loss in the furcation region of the first molars.

\section{Histometric Analysis}

The left mandibles were resected from each rat and immediately demineralized in 20\% Plank-Rychlo solution (aluminum chloride, hydrochloric acid and formic acid).

These specimens were dehydrated, embedded in paraffin, sectioned into 5 - $\mu \mathrm{m}$-thick slices along the molars in a mesiodistal direction and stained with hematoxylin and eosin. Using the image analysis system Image Tool v.3.0 (UTHSCSA, San Antonio, TX, USA), the area of periodontal ligament and/or bone loss in the furcation region of the first molars was measured in 5 semi serial sections with an interval of four sections per specimen $\left(\mathrm{mm}^{2}\right)$.

The histometric analysis showed the area limited by the bone crest and cementum surface of unligated (Figure 1) and ligated (Figure 2) teeth, as described by Nociti Jr et al. ${ }^{17}$. Measurements from the five sections were averaged to permit statistical analysis.

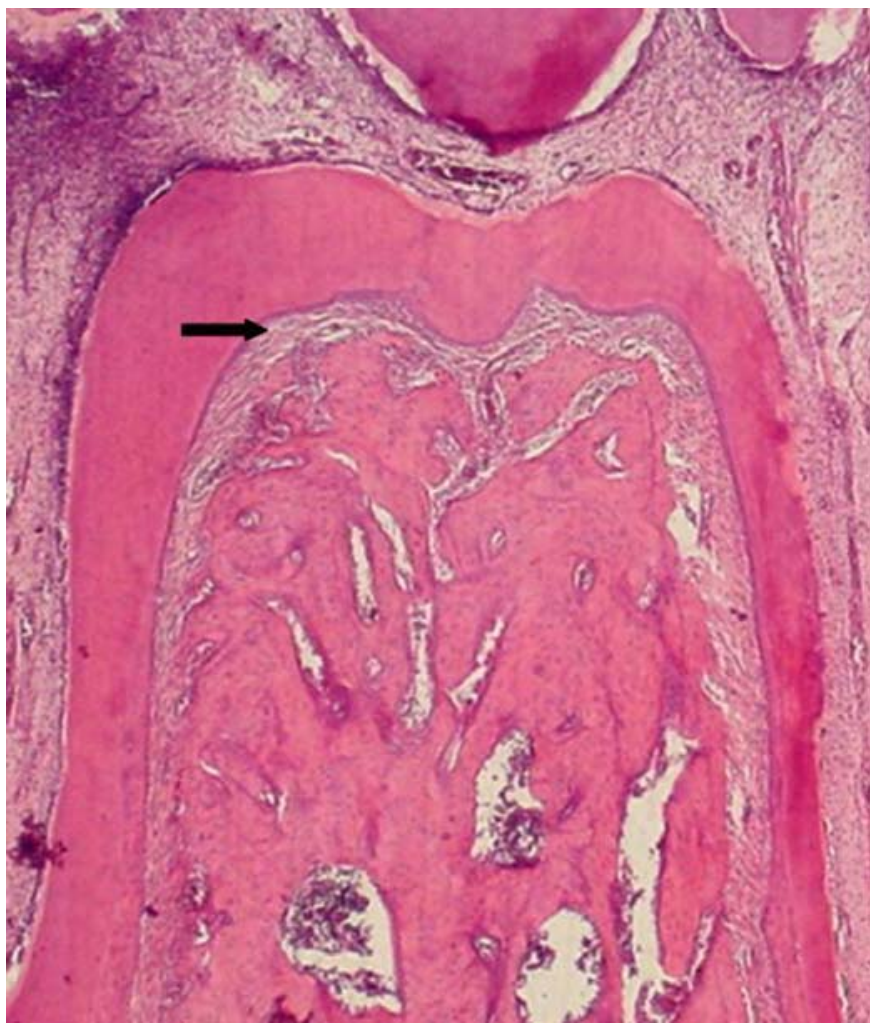

Fig. 1. Photomicrograph of the periodontal ligament area $(\rightarrow)$ in the furcation region of an unligated tooth in high ethanol group (x25 - H\&E). 
Table 1. Mean and standard deviation of analysis of diet (kcal/day) for test and control groups associated with low caloric values.

\begin{tabular}{lcc}
\hline Diet & Test A (low ethanol) & Control A (isocaloric) \\
Total calories consumed & $45.32 \pm 5.80$ & $43.64 \pm 5.72$ \\
Calories derived from protein & $34.28 \pm 4.65$ & $33.06 \pm 4.59$ \\
Calories derived from alcohol & $11.04 \pm 1.61$ & $10.58 \pm 1.67$ \\
or carbohydrate & $24.41 \pm 2.42$ & $24.35 \pm 2.61$ \\
$\%$ calories of liquid diet & $75.59 \pm 2.42$ & $75.65 \pm 2.61$ \\
\hline calories of solid diet & &
\end{tabular}

Table 2. Mean and standard deviation of analysis of diet (kcal/day) for test and control groups associated with high caloric values.

\begin{tabular}{lcc}
\hline Diet & Test B (high ethanol) & Control B (isocaloric) \\
Total calories consumed & $51.72 \pm 7.45$ & $50.27 \pm 8.10$ \\
Calories derived from protein & $31.13 \pm 5.79$ & $30.69 \pm 5.86$ \\
Calories derived from alcohol & $20.59 \pm 2.90$ & $19.58 \pm 3.51$ \\
or carbohydrate & & \\
$\%$ calories of liquid diet & $40.10 \pm 4.43$ & $39.02 \pm 4.77$ \\
$\%$ calories of solid diet & $59.90 \pm 4.43$ & $60.98 \pm 4.77$ \\
\hline
\end{tabular}

\section{Statistical analysis}

The independent sample $t$-test $(\mathrm{p}<0.05)$ was used for comparisons between differences in weigh gain and between the diets consumed in the Test and Control groups.

For histometric analyses the data were expressed as mean and standard deviation $\left(\mathrm{mm}^{2}\right)$. The independent samples $t$ test was used for comparisons between alcohol groups and control groups in presence or absence of periodontitis induced.

One-way analysis of variance and Tukey's test for subsequent multiple comparisons were used to separately determine significant differences in area of periodontal ligament and/or bone loss among the treatment groups for ligated or unligated rats. Differences were considered significant at $\mathrm{p}<0.05$.

\section{Results}

\section{Diet and Weight Analysis}

Diet analysis showed no significant differences in diet consumption $(p>0.05)$ between the Test and Control groups for low (Table 1) or high (Table 2) caloric values, considering the total number of calories consumed (kcal/day), calories derived from protein, calories derived from alcohol or carbohydrate and the percentage of calories of the liquid and solid diets.

The systemic effect of alcohol consumption observed by analysis rat's body weight showed that the ethanol feed $24 \%$ kcal per day (Test A) and pared-fed to low (Control A) ethanol consumption gained weight $(19.33 \mathrm{~g} ; \mathrm{p}=0.0000)$ during the experiment. However, no statistically significant difference was observed between baseline and final weight in the rats receiving $40 \%$ kcal per day of ethanol (Test B). Paired-fed to high (Control B) ethanol consumption lost weight.

\section{Histologic Evaluation}

Eight weeks after the beginning of the experiment, greater bone loss in the furcation region with most of the area filled for connective tissue was noted in rats that received ligature (Figure 2) compared with those without ligature (Figure 1). In the group of high caloric value of ethanol with ligature, the extent of bone loss in the furcation region was greater than in the other groups with ligature. There was a less dense periodontal ligament, characterized by the presence of smaller collagen fibers oriented horizontally to the root surface (Figure 2) and reabsorbed bone with some osteoclasts (Figure 3).

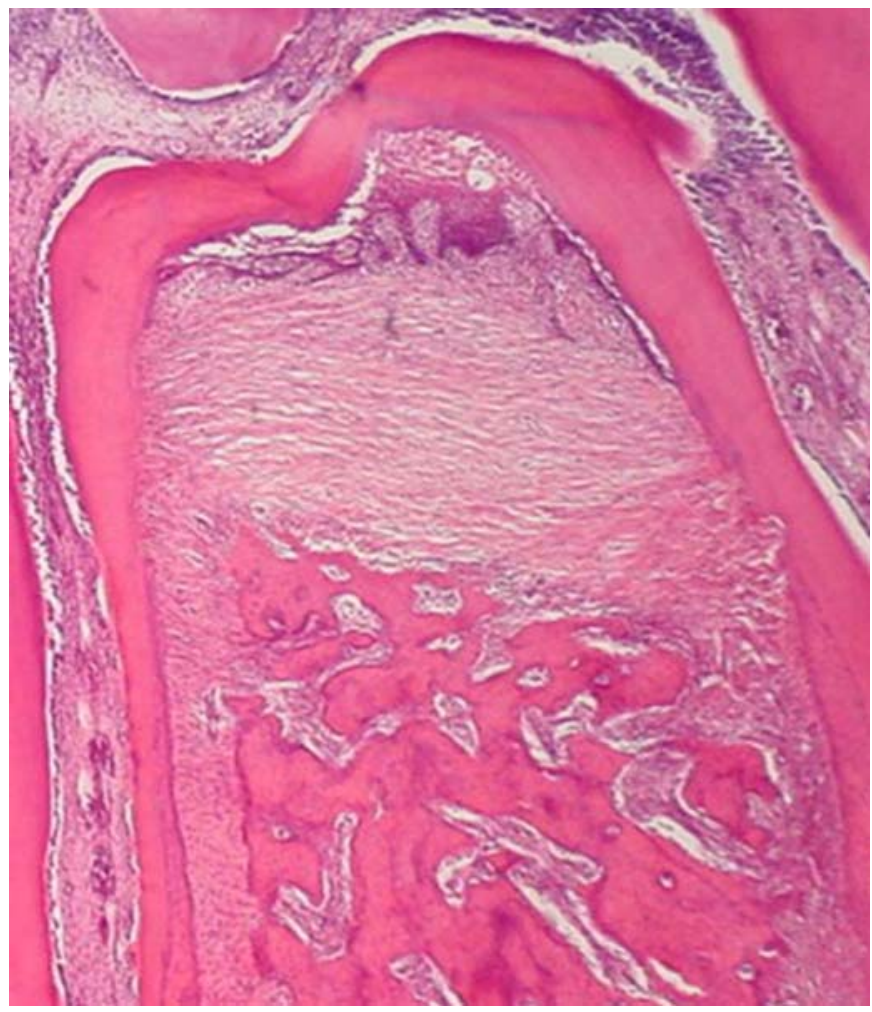

Fig. 2. Photomicrograph of the bone loss area in the furcation region of a ligated tooth in high ethanol group (x25 - H\&E). 


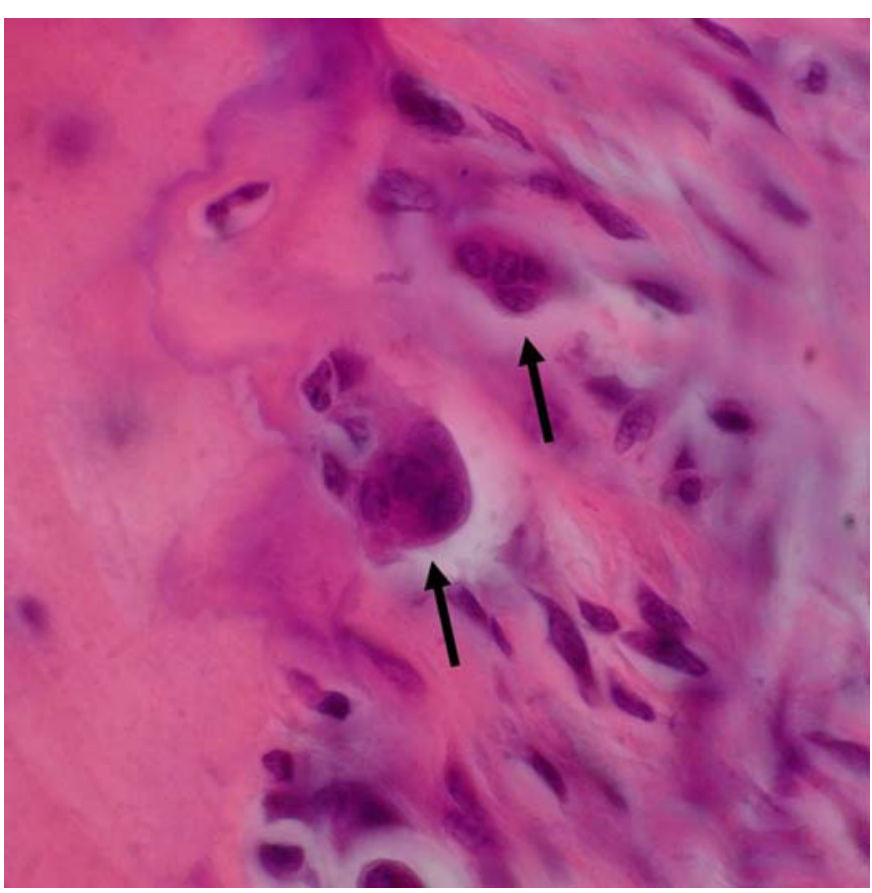

Fig. 3. Photomicrograph of the osteoclasts $(\rightarrow)$ in region of a ligated tooth in high ethanol group (X400 - H\&E).

\section{Histometric Assessment}

Histometric analysis showed that cotton ligatures placed around the teeth induced alveolar bone loss, which was confirmed by the greater bone loss $(p<0.05)$ observed in ligated teeth compared to unligated teeth.

In unligated groups, no statistically significant differences $(p>0.05)$ were observed in the periodontal ligament area among the groups Normal Control, Test A (low ethanol), Control A (low control), Test B (high ethanol) and Control B (high control). However, when ligature was placed, analysis revealed a significantly greater area of bone loss $(p<0.05)$ in the furcation region of ligated teeth in rats that received high caloric value of ethanol (Test $B$ ) than in rats receiving high caloric value of carbohydrate (Control B), low caloric value of ethanol (Test A), low caloric value of carbohydrate (Control A) and water (Normal control) (Figure 4).

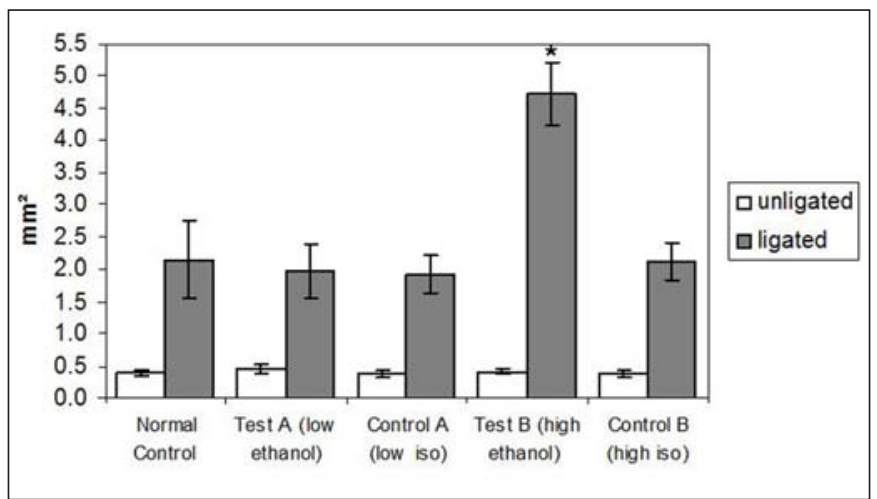

Fig. 4. Mean and standard derivation of the periodontal ligament area around unligated teeth and periodontal bone loss area around ligated teeth $\left(\mathrm{mm}^{2}\right)$ *Statistically significant (ANOVA and Tukey's test; $p<0.05$ ).
Ethanol affected ligature-induced bone loss when it represented a high value in the total of calories.

\section{Discussion}

Research suggests that alcohol consumption is associated with the increase of severity of periodontitis in humans $s^{4-5,19}$, periodontal inflammation ${ }^{14-15}$ and alveolar bone loss in periodontal disease in rats ${ }^{11-13}$. However, others studies on humans have shown no relation between periodontal disease and alcohol intake ${ }^{9-10}$.

The effects of alcohol on periodontal disease exist and this relation has been explained by biological plausibility through different mechanisms. Chronic alcoholic patients show an increased risk of developing severe infection, which may be due to altered immune response ${ }^{20}$. Alcohol has a toxic effect on the liver causing a negative effect on the clotting mechanism and frequently in alcoholic present nutritional disorders resulting from protein and vitamin deficiency ${ }^{21}$. Additionally, ethanol alters bone metabolism, as demonstrated by the dramatic effects on rat bone ${ }^{22}$. As a consequence of the toxic effects on the liver, bone, immune system and nutrition, alcohol may interfere in the mechanisms of inflammatory response in periodontal disease.

Alcohol consumption can affect the bone metabolism both directly and indirectly ${ }^{23}$. Directly, it causes the suppression of tissue formation due to toxic effect on osteoblastic activity and proliferation ${ }^{24}$, while indirectly, ethanol can aggravate bone loss as a consequence of the effects of malnutrition ${ }^{23}$

A population study that used a self-reported questionnaire regarding alcohol consumption observed a significant relationship between alcohol, gingival inflammation and clinical attachment loss, after controls to account for major confounding variables ${ }^{4}$. Another cross-sectional study ${ }^{19}$ showed that alcohol dependence might be associated with periodontal disease. This study evaluated 49 alcoholic and 49 non-alcoholic men in Brazil and observed increased severity of clinical attachment level and probing depth associated with alcohol dependence.

An important cross-sectional study involving 13,198 individuals found a moderate but consistent dose-dependent relation between alcohol consumption and increased severity of clinical attachment loss in periodontal disease ${ }^{5}$. The present study demonstrated that the effect of alcohol consumption might accentuate periodontal bone loss in ligature-induced periodontitis in female rats in a dosedependent manner. Similar findings were observed in other studies in rats ${ }^{12-13}$.

A previous study ${ }^{7}$ related alcohol consumption with periodontitis and obtained conflicting results, showing that higher alcohol consumption appears to have an inverse association with attachment loss in men, but not in women. The authors emphasized that further investigation is necessary to observe the association between alcohol and periodontitis, establishing prospective cohort studies with participants free of periodontitis at baseline. 
A longitudinal study ${ }^{6}$ that associated alcohol consumption with dental health used questions on alcohol consumption, clinical and radiographic examinations. The marginal bone level and longitudinal bone loss was determined by assessments on the proximal surfaces of all measurable teeth on radiographs from 1970 and 1990. Only teeth that were measurable in both examinations were included in the study and the results did not support an association between alcohol consumption and periodontal disease.

The reduction in human body weight during chronic alcohol consumption is associated with the fact that part of the daily diet calorie intake is provided by the alcoholic beverage ${ }^{25}$. However, this impact on weight is rarely observed in rats $^{26}$. In the present study, the systemic effect of alcohol consumption observed by analysis of rat's body weight verified that groups that consumed low ethanol or carbohydrate showed gain of weight. However, in the rats receiving high caloric values of ethanol the weight did not change and those with high carbonhydrate consumption showed weight lost. In contrast, Irie et al. ${ }^{15}$ showed a weight gain in ethanol and isocaloric groups during the experimental period associated with a high caloric value of ethanol $(36 \%)$. Analyzing rat's body weight, Souza and Rocha ${ }^{13}$ also observed that groups that consumed ethanol or carbohydrate in low or high caloric values gained weight.

Irie et al. ${ }^{15}$ and Dantas et al. ${ }^{14}$ demonstrated that ethanol consumption increased the severity of periodontal inflammation in the ligature model in rats. Irie et al. ${ }^{15}$ suggest that chronic alcohol consumption increased periodontal inflammation, oxidative damage, TNF-alpha production and had an additive effect on polymorphonuclear leukocyte infiltration and gingival oxidative damage. Dantas et al. ${ }^{14}$ suggested that ethanol consumption could represent a risk indicator for periodontal disease since augments the expression of inflammatory markers in healthy rats, and increases them significantly, at short term, in the presence of disease.

In this study, analysis of the results verified that chronic ethanol feeding may not be capable of causing bone loss in unligated rats associate with low (24\%) and high (40\%) caloric values. Souza and Rocha ${ }^{13}$ found similar findings in unligated rats associated with low $(22 \%)$ or high $(36 \%)$ caloric values of ethanol. Souza et al. ${ }^{12}$ also observed that alcohol consumption at concentrations of $10 \%$ and $20 \%$ by volume may not be capable of causing alveolar bone loss without ligature-induced periodontitis in rats. In contrast, Irie, et al. ${ }^{15}$ showed in unligated rats, that the distance between the cementoenamel junction and alveolar bone crest was greater in the ethanol group ( $36 \%$ calories derived from ethanol) than in the respective control group.

The present study demonstrated that high caloric values $(40 \%)$ of ethanol increased ligature-induced bone loss in rats. Similarly, Souza and Rocha ${ }^{13}$ showed that ethanol intake representing low caloric values ( $22 \%$ of total caloric value) aggravated bone loss in the presence of experimental periodontitis. And Souza et al. ${ }^{12}$ also evaluated rats receiving a concentration of $20 \%$ by volume showed significantly greater alveolar bone loss in ligature-induced periodontitis in female rats ${ }^{12}$. Dantas et al. ${ }^{14}$ demonstrated that short-time ethanol administration increased the length of the periodontal ligament in rats submitted to ligature induced periodontitis and diminished the alveolar bone volume, which suggest that alcohol consumption promotes the progression of this disease in the short term. Differently, Irie et al. ${ }^{15}$ reported that a high dose of ethanol itself $36 \%$ of total calories in the diet) was not capable of affecting ligature-induced bone resorption.

In conclusion, the present study demonstrated that a low-ethanol diet did not affect the periodontium, but that a high-ethanol diet may aggravate the progression of periodontitis, as demonstrated by the increased furcation region bone destruction in periodontal disease.

\section{Acknowledgements}

The present study was partially funded by the Coordination for the Improvement of Higher Education (CAPES), Brazil.

\section{References}

1. Kinane DF. Causation and pathogenesis of periodontal disease. Periodontol 2000. 2001; 25: 8-20.

2. Loe H, Anerud A, Boysen H, Morrison E. Natural history of periodontal disease in man. Rapid, moderate and no loss of attachment in Sri Lankan laborers 14 to 46 years of age. J Clin Periodontol. 1986; 13: 431-40.

3. Genco RJ. Current view of risk factors for periodontal diseases. J Periodontol. 1996; 64: 1041-9.

4. Tezal M, Grossi SG, Ho AW, Genco RJ. The effect of alcohol consumption on periodontal disease. J Periodontol. 2001; 72: 183-9.

5. Tezal M, Grossi SG, Ho AW, Genco RJ. Alcohol consumption and periodontal disease. The third national health and nutrition examination survey. J Clin Periodontol. 2004; 31: 484-8.

6. Jansson L. Association between alcohol consumption and dental health. J Clin Periodontol. 2008; 35: 379-84.

7. Kongstad J, Hvidtfeldt UA, Gronbaek M, Jontell M, Stoltze K, Holmstrup P. Amount and type of alcohol and periodontitis in the Copenhagen City Heart Study. J Clin Periodontol 2008; 35: 1032-9.

8. Weinberg MA, Bral M. Laboratory animal models in periodontology. J Clin Periodontol. 1999; 26: 335-40.

9. Amadei SU, Souza DM, Brandão AAH, Rocha RF. Influence of different durations of estrogen deficiency on alveolar bone loss in rats. Braz Oral Res. 2011; 25: 538-43.

10. Milhan NVM, Forte LFBP, Vasconcelos LMR, Balducci I, Carvalho YR. Influence of ovariectomy combined with lack of masticatory force in the evolution of periodontal disease. Braz J Oral Sci. 2014; 13: 47-52.

11. de Souza DM, Ricardo LH, Prado Mde A, Prado Fde A, Rocha RF. The effect of alcohol consumption on periodontal bone support in experimental periodontitis in rats. J Appl Oral Sci. 2006; 14: 443-7.

12. Souza DM, Ricardo LH, Kantoski KZ, Rocha RF. Influence of alcohol consumption on the alveolar bone level associated with ligature-induced periodontitis in rats. Braz Oral Res. 2009; 23: 326-32.

13. Souza DM, Rocha RF. Low caloric value of ethanol itself increases alveolar bone loss in ligature-induced periodontitis in male rats. Braz Oral Res. 2009; 23: 460-6.

14. Dantas AM, Mohn CE, Burdet B, Zorrilla Zubilete M, Mandalunis PM, Elverdin JC, et al. Ethanol consumption enhances periodontal inflammatory markers in rats. Arch Oral Biol. 2012; 57: 1211-7. 
15. Irie K, Tomofuji T, Tamaki N, Sanbe T, Eruni D, Azuma T, et al. Effects of ethanol consumption on periodontal inflammation in rats. J Dent Res. 2008; 87: 456-60.

16. Bastos MF, Gaag GLD, Romero JR, Gabrili JJM, Marques MR, Duarte PM. Effects of Cachaça, a typical Brazilian alcoholic beverage, on alveolar bone loss and density: A study in peripubertal rats. Arch Oral Biol. 2014; 59: 82-91.

17. Nociti FH Jr, Nogueira-Filho GR, Primo MT, Machado MA, Tramontina $V A$, Barros SP, et al $L_{L}$ The influence of nicotine on the bone loss rate in ligature-induced periodontitis. A histometric study in rats. J Periodontol. 2000; 71: 1460-4.

18. Vasconcelos DFP, da Silva MAD, Marques MR, Gibilini C, Vasconcelos ACCG, Barros SP. Effects of simultaneous nicotine and alcohol use in periodontitis progression in rats: A histomorphometric study. J Clin Exp Dent. 2013; 5: e95-e99.

19. Amaral $C D$, Luiz RR, Leão $A$. The relationship between alcohol dependence and periodontal disease. J Periodontol. 2008; 79: 993-8.

20. Messingham KAN, Faunce DE, Kovacs EJ. Alcohol, injury, and cellular immunity. Alcohol. 2002; 28: 137-49.

21. Lieber CS. Relationships between nutrition, alcohol use, and liver disease. Alcohol Res Health. 2003; 27: 220-31.

22. Hogan HA, Argueta F, Moe L, Nguyen LP, Sampson HW. Adult-onset alcohol consumption induces osteopenia in female rats. Alcohol Clin Exp Res. 2001; 25: 746-54.

23. Klein R.F. Alcohol-induced bone disease: impact of ethanol on osteoblast proliferation. Alcohol Clin Exp Res. 1997; 21: 392-9.

24. Gonzalez-Calvin JL, Garcia-Sanches A, Bellot V, Muñoz-Torres M, RaiaAlvares E, Salvatierra-Rios D. Mineral metabolism, osteoblastic function and bone mass in chronic alcoholism. Alcohol Alcohol. 1993; 28: 571-9.

25. Lieber CS. Relationships between nutrition, alcohol use, and liver disease. Alcohol Res Health. 2003; 27: 220-31.

26. Hefferan TE, Kennedy AM, Evans GL, Turner RT. Disuse exaggerates the detrimental effects of alcohol on cortical bone. Alcohol Clin Exp Res. 2003; $27: 111-7$. 